\title{
Ameliorative effects of Withania coagulans and Metformin on Ovarian Morphology in Rats with Polycystic Ovarian Disease
}

\author{
Hira Ayaz ${ }^{1}$, Abdul Samad ${ }^{2}$, Aiman Farogh Anjum ${ }^{3}$, Nasar Abbas Shamsi ${ }^{4}$, Sidra Arshad $^{5}$, Mahvash Khan ${ }^{6}$ \\ ${ }^{1}$ Assistant professor, Department of Physiology, Foundation University, Islamabad Pakistan \\ ${ }^{2}$ Assistant professor, Department of Physiology, Pak International Medical College, Peshawar Pakistan \\ ${ }^{3}$ Assistant professor, Department of Physiology, $\mathrm{CMH}$ Kharian Medical College, Kharian Cantonment Pakistan \\ ${ }^{4}$ Assistant professor, Department of Physiology, Foundation University, Islamabad Pakistan \\ ${ }^{5}$ Associate professor, Department of Physiology, Rawal Institute of Health Sciences, Islamabad Pakistan \\ ${ }^{6}$ Professor, Department of Physiology, Rawal Institute of Health Sciences, Islamabad Pakistan
}

\begin{abstract}
AB S TR ACT
Background: Various medicinal herb plants are being used in place of metformin for treatment of polycystic ovarian disease for their less harmful effects. Withania coagulans (WC) is a herb known for its insulin sensitizing and weight reducing properties. The present study was done to determine the influence of aqueous extract of Withania coagulans (aqWC) and metformin on ovarian weight and ovarian folliculogenesis in polycystic ovarian disease-induced rats.

Material and Methods: An experimental animal study was carried out at the Physiology Department of Islamic International Medical College, Rawalpindi from April 2016 to March 2017. Forty female Sprague Dawley rats were divided initially into two groups. Group A (Normal Control) and Disease induced group. Standardized laboratory diet was fed to Group A while the disease induced group was given standardized laboratory diet and letrozole solu tion orally $(1 \mathrm{mg} / \mathrm{kg})$ for 21 days to induce Polycystic ovary syndrome, which was established by observing estrous cycle of rats. Disease induced group was then split into group B (PCOS control), C (Aqueous Withania coagulans treated) and D (Metformin treated) and observed after 14 days. Groups A and B underwent ovarian dissection after 21 days and groups $C$ and $D$ underwent dissection at the end of experiment (after 35 days). Independent sample $t$-test was used for the comparison between the control and disease induced group.

Results: Group B showed a significant increase in ovarian weight in comparison to group A rats $(P<.05)$. Treatment with Withania coagulans and metformin significantly decreased ovarian weight $(P<.05)$ and increased primary, Graafian, antral follicular count and corpus luteum along with reduction in cystic follicular count in letrozole -induced polycystic ovarian disease rats. Improved folliculogenesis was also observed in the same groups (groups C \& D).

Conclusions: Withania coagulans can be a substitute for improvement of ovarian follicular development in polycystic ovarian disease.

Key words: Estrous cycle, Metformin, Polycystic ovarian disease, Withania coagulans.

Authors' Contribution:

${ }^{1}$ Conception; Literature research; manuscript design and drafting; ${ }^{2,3}$ Critical analysis and manuscript review; ${ }^{4-6}$ Data analysis; Manuscript Editing.

Correspondence: Article info:

Hira Ayaz

Email:hiraayaz6@gmail.com

Received: November 11, 2019

Accepted: February 22, 2021
\end{abstract}

Cite this article. Ayaz H, Samad A, Anjum AF, Shamsi NA, Arshad S, Khan M. Ameliorative effects of Withania coagulans and Metformin on Ovarian Morphology in Rats with Polycystic Ovarian Disease. J Islamabad Med Dental Coll. 2021; 10(1): 31-36. Doi:

Funding Source: Nil

Conflict of Interest: Nil 


\section{Introduction}

Polycystic ovary syndrome (PCOS) is a globally recognized reproductive endocrine disorder and a principal cause of anovulatory infertility affecting 8 to $13 \%$ of women of reproductive age. Anovulation is recognized as a disorder in the development of follicles and exists in $70 \%$ of females having PCOS. ${ }^{1}$ The dynamics of early follicular development in PCOS are different from normal ovarian folliculogenesis. Different studies have shown that decreased amounts of oocyte-secreted growth factors, androgen overproduction and increased responsiveness to Luteinizing hormone (LH) and hyperinsulinemia give rise to production of large number of small sized antral follicles in early stage. These small follicles lead to higher anti-müllerian hormone (AMH) levels, which reduce the follicular sensitivity to FSH due to which antral follicles cannot develop into mature follicles and undergo ovulation. As a result, excessive early follicular growth and failure of maturation of one follicle from the pool of follicles into a dominant follicle (under the influence of $L H$ ) takes place. This is known as follicular arrest, the main ovulatory disorder of PCOS. ${ }^{2,3}$

Management of infertility in PCOS involves bringing about weight loss in obese, infertile women, inducing ovulation by use of clomiphene citrate and increasing insulin sensitivity by use of metformin. Various studies have shown that metformin improves ovulation rate and clinical pregnancy rate. ${ }^{4,5}$ But continuous administration of metformin at high doses has its own hazardous effects. Some patients also have reduced compliance to metformin due to its adverse effects (gastrointestinal upset, lactic acidosis and vitamin B12 deficiency), because of which its usage is limited or contraindicated in those cases. ${ }^{6,7}$

In place of metformin various kind of medicinal herb plants have been in use due to absence of side effects, easy availability and affordability. One of these herbs is Withania coagulans dunal, also known as Paneer doda, commonly found in North India, Afghanistan and Pakistan. It is well known for its insulin sensitizing features because of Withanolides present in it. ${ }^{8,9,10}$ Due to its known effects as antidiabetic and insulin sensitizer herb, it might help in reducing the severity of PCOS by improving glucose intolerance and insulin resistance leading to increased fertility rate.

The aim of this study was to investigate the effects of aqueous extract of Withania coagulans (aqWC) and Metformin on ovarian weight and morphology in PCOS rats. By comparing the effects of WC and metformin, we might be able to identify whether Withania coagulans can be substituted for metformin in alleviation of symptoms of PCOS or not.

\section{Material and Methods}

This experimental animal study was conducted at the Multidisciplinary research laboratory of Islamic International Medical College, Riphah International University in collaboration with National Institute of Health (NIH), Islamabad Pakistan from April 2016 to March 2017. It was conducted after getting approval from Ethics Review Committee of Islamic International Medical College (Ref \# Riphah/IIMC/ERC/16/0119).

Forty female Sprague Dawley rats weighing 180-250 $\mathrm{g}$ were acquired from NIH (Islamabad, Pakistan). Rats aged 6-8 weeks were included in the study as they attain sexual maturity at this age. Pregnant rats and those having any obvious physical deformity were excluded from the study. The animals were kept at a temperature of $24^{\circ} \mathrm{C}$, humidity of $50 \%$ with a 12-hour light and dark cycle. The study rats $(n=40)$ 
were randomly distributed into two groups; Group A serving as normal control $(n=10)$ and Diseaseinduced group $(n=30)$. Standardized laboratory diet was given to group $\mathrm{A}$, while standardized laboratory diet and Letrozole (trade name Lezra) $1 \mathrm{mg} / \mathrm{kg}$, mixed in $0.5 \%$ carboxy methyl cellulose (CMC) 2 $\mathrm{mg} / \mathrm{kg}$, orally for 21 days to induce PCOS were administered to the Disease induced group.

The Disease induced group was then split into group B (PCOS control) $(n=10)$, group C (Withania coagulans-treated) $(n=10)$ and D (Metformintreated) $(n=10)$.

From nearby local market desiccated fruits of Withania coagulans were acquired and used after getting recognized and certified by National Agriculture Research Center (NARC), Islamabad (Herbarium section - voucher number IIMC 03). Dried fruit of Withania coagulans (1 kg) was crumpled after the removal of calyx and pedicles and soaked overnight in $4 \mathrm{~L}$ of purified water at moderate temperature. On the next day, the softened extract was sieved by using filter paper. After the process of filtration, water extract was evaporated at $55-60^{\circ} \mathrm{C}$ on a magnetic stirrer evaporator for 7 hours to get a semi-solid material (yield 16\% wt/wt). Later on, this yield at a dose of $250 \mathrm{mg} / \mathrm{ml}$ was diluted in purified water. An aqueous extract of $W C, 1000 \mathrm{mg} / \mathrm{kg} /$ day was dissolved in drinking water and fed orally to group $C$ rats along with standardized laboratory diet.

Metformin (Glucophage) was purchased from D. Watson pharmacy, Rawalpindi, Pakistan. Metformin tablet ( $500 \mathrm{mg}$ ) was dissolved in $25 \mathrm{ml}$ of normal saline to prepare a solution of $2 \mathrm{mg} / 100 \mathrm{ml}$. The solution was kept covered by aluminum foil and stored at $4^{\circ} \mathrm{C}$. Metformin solution mixed in drinking water and standardized laboratory diet was given to group $D$ for 14 days. Vaginal smears were taken from the rats by swab smear technique for 21 days sequentially, to observe the phase of the estrus cycle and confirm PCOS induction. If any phase of this cycle persisted continously for more than 3 days it showed irregularity in the cycle. ${ }^{10,11,12}$ Rats with irregular ovarian cycles (Diestrus phase for 3 consecutive days) were considered as PCOS positive and were selected for the trial. On $22^{\text {nd }}$ day, animals in group $A$ and Disease-induced group were sacrificed by cervical dislocation. After dissection, ovaries were removed, fat cleaned off and weighed on a scale (TS200 electronic compact scale, Jiangyin Ditai electronic technology Co. Ltd., China). At the time of removal, ovaries of rats in Disease induced group were in diestrus phase whereas in groups $A, C$ and $D$, the ovaries were in proestrus phase. Ovaries were fixed in $4 \%$ paraformaldehyde in phosphatebuffered saline (PBS) for 1 hour at room temperature, soaked in PBS, dried and embedded in paraffin. For hematoxylin and eosin (H\& E) staining, $4 \mu \mathrm{m}$ tissue sections of ovaries were prepared. Tissue sections were deparaffinized using xylene, hydrated through an ethanol series of $100 \%, 90 \%$, $80 \%$ and stained with H\&E. Sections were then mounted with Canada balsam after washing and observed under light microscope using x100 lenses. The primary follicles, antral follicles, Graafian follicles and corpus luteum per ovary were identified and determined by counting 5 representative sections per ovary at least $20 \mu \mathrm{m}$ apart.

Statistical analysis was performed by applying the Statistical Package for Social Sciences version 21 (SPSS 21). Independent sample t-test was used for the comparison between the control and disease induced group. Results were represented as mean \pm SEM. For both analyses, $P$-value $<.05$ was taken as significant.

\section{Results}

Group B showed a significant increase in ovarian weight in comparison to group $A$ rats $(P<.05)$. Rats in groups $C$ and $D$ showed a significant reduction $(P$ 
$<.05)$ in ovarian weight in comparison to group B as displayed in Table I.

Table I: Comparison of Mean \pm SEM of ovarian weight in rats in all four groups

\begin{tabular}{|l|c|}
\hline Groups & Ovarian weight \\
\hline Group A & $57.20 \pm 0.83$ \\
\hline Group B & $64.68 \pm 0.53^{*}$ \\
\hline Group C & $61.60 \pm 0.43^{*}$ \\
\hline Group D & $58.54 \pm 0.19^{*}$ \\
\hline
\end{tabular}

${ }^{*} P$-value $<.05$ was considered statistically significant

The average count of primary, antral and Graafian follicles and corpus luteum was significantly reduced $(P<.05)$ in group $B$ as compared to group $A$, whereas the cystic follicular number was significantly increased $(P<.05)$ in group B than group A. Groups $C$ and $D$ depicted significant increase $(P<.05)$ in primary, antral and Graafian follicles and corpus luteum in contrast to group B. Cystic follicles were significantly reduced in groups $C$ and $D$ as compared to group B (Table II).

Rats in group $D$ showed significant decrease $(P<.05)$ in ovarian weight in comparison to group $C$. However, no significant results were observed in the count of primary follicle while comparing rats in group $C$ and D. Group D rats depicted significant increase $(P<0.05)$ in antral follicles, Graafian follicles and corpus luteum in contrast to group $C$. Cystic follicular count showed significant reduction $(P<.05)$ in rats of group $D$ in contrast to group $C$.

Histological analysis of Group A showed several phases of development of follicles such as primary follicles (PF), antral follicles (AF), Graafian follicle (GF) and Corpus luteum (CL) as shown in Figure 1. Group $B$ rats showed major differences than group $A$, as small-sized Primary follicles and Antral follicles were seen along with appearance of large cystic follicles (CF). No Graafian follicle and Corpus luteum were seen. Morphological analysis of ovaries of rats in group $C$ and $D$ presented improved folliculogenesis than group $B$, showing $P F, A F, G F$ and $\mathrm{CL}$ (Figure 1).

\section{Discussion}

In this study, ovarian weight and morphology of the rats treated with Withania coagulans and metformin were compared with Disease-induced rats (PCOSinduced rats). The results showed that administration of aqueous extract of Withania coagulans decreased ovarian weight and improved folliculogenesis as well. This effect might be due to the presence of active components, known as Withanolides in aqWC. The most likely mechanism is that the withanolides increase the tissue receptivity to insulin, which then amends the gonadotropin levels.

In the current study, effect of Withania coagulans and metformin on ovarian weight $(\mathrm{mg}$ ) and ovarian morphology (number and type of follicles) was assessed along with their comparison. Findings of the present-daystudy are in concurrence with the study conducted by Rajan et al. inspecting the consequence of Soy isoflavones $(100 \mathrm{mg} / \mathrm{kg})$ administration for 14 days on body weight, ovarian and uterine weight in letrozole $(1 \mathrm{mg} / \mathrm{kg})$ induced PCOS rats. They revealed that Soy isoflavones $(100 \mathrm{mg} / \mathrm{kg})$ treatment significantly improved the letrozole-induced PCOS symptoms by bringing about a decrease in ovarian, uterine and general body weight. They also proposed that these weight lowering effects might be due to the presence of phytoestrogens in isoflavones. ${ }^{13}$ This study differed from our study as they administered two different doses of soy isoflavones and observed their effects on symptoms of PCOS whereas we treated the rats with aqWC and metformin and compared their effects on PCOS-induced rats.

Another study by Bhutani et al. observed that administration of aqueous extract of Symplocos racemosa Roxb. $(2000 \mathrm{mg} / \mathrm{kg}$ ) for 6 days resulted in reinforcement of folliculogenesis in ovaries. ${ }^{14}$ These results were same as witnessed in the existing study though immature rats were not chosen. 


\begin{tabular}{|l|c|c|c|c|}
\hline \multicolumn{4}{|c|}{ Table II: Comparison of mean \pm SEM of different follicles in ovaries of rats in all four groups } \\
\hline Type of Follicle & Group A & Group B & Group C & Group D \\
\hline Primary follicle & $5.8 \pm 0.92$ & $4.0 \pm 0.47^{*}$ & $5.4 \pm 0.33^{*}$ & $5.6 \pm 0.45^{*}$ \\
\hline Antral follicle & $3.9 \pm 0.27$ & $2.8 \pm 0.49^{*}$ & $3.4 \pm 0.27^{*}$ & $4.1 \pm 0.26^{*}$ \\
\hline Graafian follicle & $3.7 \pm 0.23$ & $0.0 \pm 0.00^{*}$ & $2.0 \pm 0.24^{*}$ & $2.2 \pm 0.25^{*}$ \\
\hline Corpus luteum & $3.4 \pm 0.16$ & $0.0 \pm 0.00^{*}$ & $1.0 \pm 0.26^{*}$ & $2.0 \pm 0.51^{*}$ \\
\hline Cystic follicle & $0.0 \pm 0.00$ & $4.0 \pm 0.47^{*}$ & $1.5 \pm 0.42^{*}$ & $0.5 \pm 0.70^{*}$ \\
\hline
\end{tabular}

$* P<.05$ is considered statistically significant.

\begin{tabular}{|l|c|c|c|c|c|c|}
\hline \multicolumn{6}{|c|}{ Table III: Comparison of mean \pm SEM of ovarian weight and follicular count between rats in Groups C and D } \\
\hline Groups & $\begin{array}{c}\text { Ovarian } \\
\text { weight } \mathbf{( m g )}\end{array}$ & $\begin{array}{c}\text { Primary } \\
\text { follicle }\end{array}$ & $\begin{array}{c}\text { Antral } \\
\text { follicle }\end{array}$ & $\begin{array}{c}\text { Graafian } \\
\text { follicle }\end{array}$ & $\begin{array}{c}\text { Corpus } \\
\text { luteum }\end{array}$ & Cystic follicle \\
\hline Group C & $61.4 \pm 0.34^{*}$ & $5.4 \pm 0.20$ & $3.42 \pm 0.02^{*}$ & $2.03 \pm 0.05^{*}$ & $1.23 \pm 0.25^{*}$ & $1.53 \pm 0.05^{*}$ \\
\hline Group D & $58.3 \pm 0.22^{*}$ & $5.7 \pm 0.10$ & $4.19 \pm 0.10^{*}$ & $2.26 \pm 0.11^{*}$ & $2.03 \pm 0.05^{*}$ & $0.53 \pm 0.57^{*}$ \\
\hline
\end{tabular}

$* P<.05$ is considered statistically significant.
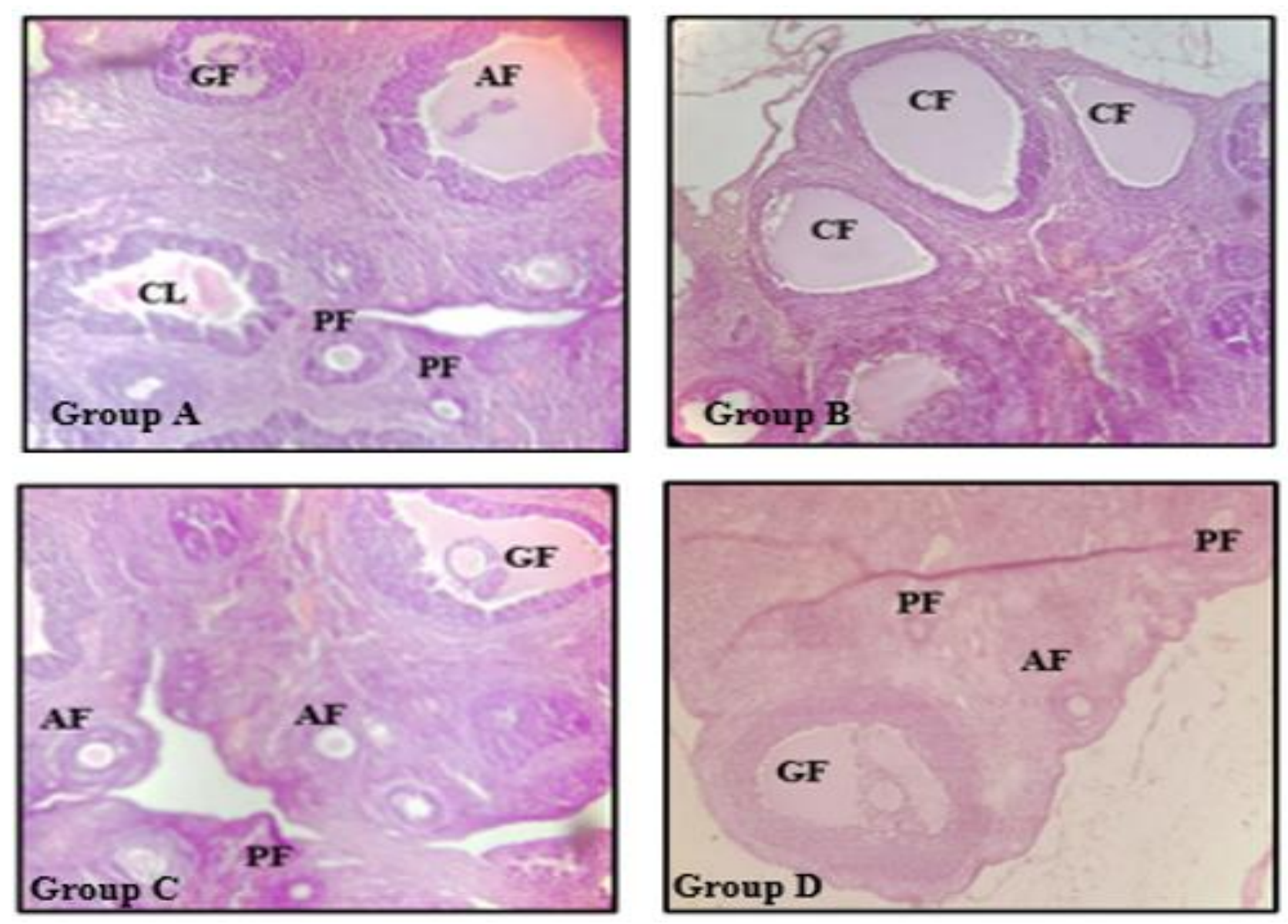

Figure 1: Photomicrographs of ovarian tissue in Groups A, B, C and D (H\&E; x100).

We used letrozole-induced PCOS rats and also showed a comparison to metformin treatment.

Results reported by Hojatullah et al. also witnessed restoration of folliculogenesis in estradiol valerate induced PCOS rats after administration of palm pollen extract $(400 \mathrm{mg} / \mathrm{kg})$ for 21 days. They proposed that glycosidal, flavonoids and saponins present in extract are responsible for antioxidant properties of palm pollen extract resulting in reestablishment of normal ovarian functions. ${ }^{15}$ Similar 
results were observed in the present study but letrozole induced animal model with only a single dose of aqueous extract of aqWC was used.

\section{Conclusion}

Notable ameliorative potential of Withania coagulans observed in this study illustrates that it may be used as a substitute for metformin in ameliorating ovarian histological features in PCOS.

\section{Acknowledgment}

We acknowledge the help and guidance provided by Mr Hussain, Incharge of animal house at National Institute of Health, Islamabad during this project.

\section{References}

1. Neven AC, Laven J, Teede HJ, Boyle JA. A summary on polycystic ovary syndrome: diagnostic criteria, prevalence, clinical manifestations, and management according to the latest international guidelines. Semin Reprod Med. 2018; 36(1): 5-12. Doi: 10.1055/s-00381668085.

2. Qiao J, Feng HL. Extra-and intra-ovarian factors in polycystic ovary syndrome: impact on oocyte maturation and embryo developmental competence. Hum Reprod. 2011; 17(1): 17-33. Doi: 10.1093/humupd/dmq032.

3. Franks S, Hardy K. What causes anovulation in polycystic ovary syndrome? Curr Opinion Endocr Metab Res. 2020; 12: 59-65. Doi: 10.1016/j.coemr.2020.03.001.

4. Sirmans SM, Pate KA. Epidemiology, diagnosis, and management of polycystic ovary syndrome. Clin Epidemiol. 2013; 6: 1-13. Doi: 10.2147/CLEP.S37559.

5. Sam S, Ehrmann DA. Metformin therapy for the reproductive and metabolic consequences of polycystic ovary syndrome. Diabetologia. 2017; 60(9): 1656-61. Doi: 10.1007/s00125-017-4306-3.

6. Practice Committee of the American Society for Reproductive Medicine. Role of metformin for ovulation induction in infertile patients with polycystic ovary syndrome (PCOS): a guideline. Fertil Steril. 2017; 108(3): 426-41. Doi: 10.1016/j.fertnstert.2017.06.026.

7. Urbano J, Fernandes AC, Ferreira P, Pimenta J. Severe neuropsychiatric symptoms due to vitamin b12 deficiency: a case of pernicious anemia or metformin use? Galicia Clinica. 2015;76(4):178-80.

8. Peerzade N,Sayed N, Das N. Antimicrobial and phytochemical screening of methanolic fruit extract of Withania coagulans L Dunal for evaluating the antidiabetic activity. Pharma Innovation J. 2018; 7(1): 197-204.

9. Upadhayay A, Shalini S, Kumari S, Rahman MU. Evaluation of antidiabetic activity of fruits of withahnia coagulans in streptozocin induced diabetic rats. J Drug Deliv Ther. 2018; 8(2): 25-8.

10. Samad A, Rajpoot NN, Ayaz H, Sadiq N. Effect of Withania coagulans and Liraglutide on Serum Glp-1, Postprandial and Fasting Blood Glucose in Streptozotocin Induced Diabetic Rats. JBUMDC. 2019: 120.

11. McLean, A. C., Valenzuela, N., Fai, S., Bennett, S. A. L. Performing Vaginal Lavage, Crystal Violet Staining, and Vaginal Cytological Evaluation for Mouse Estrous Cycle Staging Identification. J Vis Exp. 2012; 67: e4389. Doi: 10.3791/4389.

12. Westwood FR. The female rat reproductive cycle: a practical histological guide to staging. Toxicol Pathol. 2008; 36(3): 375-84. Doi: 10.1177/019262330 8315665.

13. Gulinello M. Behavioral Core Protocols and Training. Elevated Plus Maze. Albert Einstein College of Medicine: Behavioral Core Facility. 2016.

14. Rajan RK, Balaji B. Soy isoflavones exert beneficial effects on letrozole-induced rat polycystic ovary syndrome (PCOS) model through anti-androgenic mechanism. Pharm Biol. 2017; 55(1):242-251. Doi: 10.1080/13880209.2016.1258425.

15. Jadhav M, Menon S, Shailajan S. Anti-androgenic effect of Symplocos racemosa Roxb. against letrozole induced polycystic ovary using rat model. J Coastal Life Med. 2013; 1(4): 309-14. Doi: 10.12980/JCLM.1.2013C79.

16. Karimi Jashni $H$, Kargar Jahromi $H$, Bagheri Z. The effect of palm pollen extract on polycystic ovary syndrome (POS) in rats. Int J Med Res Health Sci. 2016; 5(5): 317-21. 